This work is licensed under a Creative Commons Attribution 4.0 International License https://creativecommons.org/licenses/by/4.0/

\title{
PRAGMATIC MARKER I PRAY IN MIDDLE ENGLISH WRITTEN MONUMENTS
}

\author{
Natalia Yu. Merkuryeva \\ Moscow Metropolitan Governance Yury Luzhkov University, \\ 28, Sretenka st., Moscow, 107045, Russia
}

\begin{abstract}
The article deals with the variations of predicative pragmatic marker I pray yow / thee. The linguistic material is taken from the texts that were written in XIV-XV centuries by English authors and created in different genres (plays, ballades, visions, confessions, etc.) containing elements of dialogic speech. It has been found that the marker was not a stiff structure during the period under consideration. The variations of the expression were formed as the result of miscellaneous processes that can be seen in its following features: non-fixed composition of the marker, that can be enlarged (by an adverb here I the pray, a modal verb I wold the pray) and can be reduced (by elision of a object pronoun I preye), non-permanent word order depending on the requirement of a rhyme and preferences of the writer (I pe praye, y pray $P e$, preye I thee), the representation of the expression in the sentence as both parenthetical clause and main one (followed by a that-subordinate clause). The peculiarities of the location of the marker in the sentence are discussed. If I pray yow/thee is the only one pragmatic marker in the sentence, it is placed at the beginning or at the end of it. The placement of the parenthetical expression inside the receiving sentence is characteristic of the individual style of the author and is not common. If the expression is combined with other markers, predicative and non predicative, there are two variants of their localization in the sentence: "pointwise», when several markers are concentrated at the beginning or at the end of the construction, and "framework», when phatic parts are located in ante- and postposition of the markers receiving sentence.
\end{abstract}

Key Words: pragmatic markers, discourse markers, historical syntax, predicative markers, authorial style

\section{ПРАГМАТИЧЕСКИЙ МАРКЕР I PRAY В СРЕДНЕАНГЛИЙСКИХ ПИСЬМЕННЫХ ПАМЯТНИКАХ}

\author{
Н.Ю. Меркурьева
}

Московский городской университет управления Правительства Москвы имени Ю.М. Лужкова, 107045, Россия, Москва, ул. Сретенка, 28 
Аннотация. В статье рассматриваются разновидности предикативного прагматического маркера I pray yow / theе. В качестве источника лингвистического материала использованы произведения английских авторов, созданные в XIV-XV вв. и содержашие элементы диалогической речи (пьесы, баллады, видения, исповеди и др.). Выявлено, что на изучаемом этапе оборот не является застывшей структурой. Разновидности оборота формируются как результат разнообразньх проиессов - расширения (за счёт наречия here I the pray, модального глагола I wold the pray) и сужения (при редукиии местоимения-дополнения I ргеуе), изменения порядка слов под влиянием требований рифмы или предпочтений автора (I pe praye, y pray Pe, preye I thee), оформления оборота в предложении (в качестве вводной клауземы или главной, принимающеей that-придаточную). Обсуждаются особенности расположения оборота в предложении. Если I рray yоw / theе является единственным прагматическим маркером, он размещается в начале или бинале конструкиии; вклинивание в принимающее предложение не типично и характеризует индивидуальныи стиль автора. При сочетании оборота с другими прагматическими маркерами, предикативными и непредикативными, наблюдаются два варианта их размещения в предложении: «точечно», скониентрацией нескольких маркеров в начале или финале конструкиии, и «рамочно», сразмещением фатических составляющих в пре- и постпозиции от принимающего маркеры предложения.

Ключевые слова: прагматические маркеры, дискурсивные маркерь, маркерь предикативного типа, исторический синтаксис, авторский стиль

\section{Введение}

$\mathrm{K}$ ак известно, прагматические маркеры (дискурсивные маркеры, дискурсивные слова, прагматемы, прагматические операторы, дискурсивные частицы) - широкий класс языковых единиц, представленный словами, фразами, звуками, не имеющими реального лексического значения и привлекаемые говорящим для формирования разговорной структуры. К прагматическим маркерам относят междометия (oh, well), модально-предикативные сочетания (as I say, you know, I mean, I think), фразы (to begin with, of course, by the way), наречия (right, particularly, so, then), восклицания (yes, okay), союзы (and, or, because) и др. Разнообразие и функционирование прагматических структур в речи разных стилей разных языков на современном этапе их развития тщательно изучается $[13 ; 14 ; 15 ; 18]$.

Прагматическим маркерам присуща полифункциональность, что признаётся всеми исследователями. Маркеры организуют текст, выполняя структурную и смысловую функции. С их помощью реализуются межличностные отношения и уточняется их характер, может быть снижена, а в ряде случаев и усилена, конфликтогенность. Прагматические маркеры употребляются для уточнения полученной информации и расширения её объёма, эмоционального усиления высказывания, создания определённой тональности. Они придают тексту динамику, модифицируя его в коммуникативное событие [11, с. 169]. Особенно широко маркеры употребляются в устном дискурсе. Свободно встраиваясь в спонтанную устную речь, они проговариваются машинально, без предварительного обдумывания, на уровне речевого автоматизма. Обращение к прагматическим маркерам особо частотно в целях заполнения паузы хезитации, возникающей при поиске последующего слова в речевой цепи или осмыслении говорящим структуры собственной речи [5, с. 388]. Привлечение разных дискурсивных маркеров обеспечивает нормальное функционирование коммуникативного взаимодействия, маркеры являются теми необходимыми средствами, которые обеспечивают речевую коммуникацию [4, с. 120]. Обладая свойством полифункциональности, маркеры объединяются общим назначением - служат связующим элементом между говорящим, слушающим, местом и временем, обеспечивают связность текста, организуют последовательность высказываний, показывают отношения между сегментами дискурса [17, p. 332]. 
Основное внимание исследователей уделяется прагматическим маркерам в языке и речи современного этапа. Функционированию прагматических маркеров на протяжении других исторических периодов уделяется гораздо меньшее внимание.

В настоящей работе обратимся к периоду XIV-XV вв. - чрезвычайно значимой эпохе в истории английского языка. Считается, что именно в этот период особенно интенсивно происходило формирование национального литературного языка, который возвысился над местными диалектами, становясь способом общения всей нации [10, с. 140]. Наиболее яркими событиями богат XIV век: в 1349 году было предложено вести преподавание латинского языка не на французском, а на английском языке, в 1362 году король обратился к парламенту на английском языке, и с тех пор тронную речь произносили на этом языке. В том же 1362 году парламент выпустил решение о рассмотрении судебных дел на английском языке, а с 1363 года вместо французского языка в парламентских прениях стал использоваться английский. Столетием позже, начиная примерно с 1477 года английский стал языком школьного преподавания [2, с. 26; 16, p. 417-418].

Жанры, к которым обращаются авторы XIV-XV вв., разнообразны - рыцарские поэмы и баллады, аллегорические поэмы и трактаты, проповеди и поэмы-видения. Большинство произведений представляет собой «синтетические жанровые образования», то есть имеет неоднородную жанровую структуру, при этом жанры видения, паломничества, исповеди и дебата (словопрения) являются господствующими [8, с. 12]. Так, например, в «Кентерберийских рассказах» Чосера выделяются рыцарский роман, благочестивая христианская легенда, новелла, проповедь, животный эпос, трактат и другие, рассказчики разыгрывают интермедии, которые напоминают жанровые ситуации словопрения и моралите [там же, с. 13-14]. Считается, что на начальных этапах литературному языку была чужда функция обиходно-бытового общения, сам характер текстов был далёк от разговорного языка [12, с. 192]. Однако, если авторы, создавая образы действующих лиц, прибегали к употреблению прямой речи, они включали в реплики своих персонажей определённые элементы живой разговорной речи в целях придания описываемым ситуациям правдоподобия и сходства с реальными событиями. Можно предположить, что такими элементами были прежде всего прагматические маркеры.

При проведении исследования предстояло найти и проанализировать тексты, содержащие фрагменты диалогической речи, и рассмотреть наиболее часто встречающиеся прагматические маркеры, использовавшиеся авторами, а, значит, с большой степенью вероятности находившиеся в речевом обиходе создателя текста и его современников. Одним из таких маркеров оказался оборот, образованный глаголом pray.

Глагол preien, имеющий значения как светское ask earnestly, beg (someone), так и религиозное pray to a god or saint, впервые отмечен в текстах начала XIII века. Он произошёл от старофранцузского preier, означающего to pray, в старофранцузский же слово preier пришло из латыни в виде слов precari (в вульгарной латыни - форма precare) со значениями ask earnestly, beg, entreat и prex (с формами множественного числа preces, родительного падежа precis), со значением prayer, request, entreaty. В составе вводного предложения глагол pray появился позже и впервые, в форме I pray thee, засвидетельствован в текстах конца XIV века [9]. Именно этим обусловлен выбор временных рамок исследования. Оборот считается синонимом для прагматических маркеров please и if you will, придающим всей фразе оттенок почтительности, что нашло отражение в его русских эквивалентах - “пожалуйста”, “прошу вас!” [7, с. 577], “ну, пожалуйста”, “ради бога”, “скажите на милость”, “очень прошу” [3, с. 199]. Рассмотрим некоторые структурные и стилистические особенности, которые были свойственны сочетанию I pray thee на начальном этапе функционирования, в XIV-XV веках.

\section{Материалы и методы}

Источником лингвистического материала послужили произведения английских авторов, написанные в XIV - XV вв. Особый интерес в плане поисков элементов разговорной речи представляли мистерии - средневековые пьесы, основанные на религиозных сюжетах: "Мистические 
пьесы Таунли”/ “The Towneley Plays”, написанные в первой половине XIV века на северном диалекте; “Йоркские мистерии” /“York Plays”, написанные в первой половине XV века (сохранившийся манускрипт датируется 1463-1477 гг.), также представляющие северный диалект [1, с. 136]; "N-Town Plays", написанные во второй половине XV века на восточно-центральном диалекте. Необходимый для исследования материал был найден в тексте “Кентерберийских рассказов” Джеффри Чосера, созданных в период 1387-1400 гг. и представляющих Лондонский диалект, а также в ряде других произведений XIVв. - в поэме “Сэр Гавейн и зелёный рыцарь” /“Sir Gawain and the Green Knight” на западно-центральном диалекте, в одной из версий легенды о Гае из Уорика, принадлежащей перу неизвестного автора; поэме Джона Гауэра “Исповедь влюбленного” /“Confessio Amantis”, датируемой 1386-1390 гг. Для исследования применялись методы проведения сплошной выборки изучаемого материала, лингвистического исследования языковых фактов, лингвистического описания, сравнительно-сопоставительного анализа.

\section{Результаты и обсуждение}

Как известно, в среднеанглийском языке только намечается тенденция к выработке устойчивого порядка слов, современный твёрдый порядок слов окончательно установился лишь в конце XVII в., то есть примерно к концу ранненовоанглийского периода [2, с. 208; 6, с. 270]. Неустойчивость языковых норм просматривается и в изучаемой структуре. В этой связи для проведения анализа представлялось необходимым принять определённые элементы за «обязательные» составляющие оборота. Такими «обязательными» словами будем считать подлежащее $I(Y)$, сказуемое pray (записанное также в виде praye и preye, prey, в последних двух вариантах просматривается старофранцузский корень preier) и дополнение yow или the (the, pe). Тогда другие присутствующие в обороте элементы примем за вариативные, и структуры, имеющие разного рода расхождения с «обязательным» составом, будем рассматривать как разновидности оборота.

В первую очередь обратимся к лексико-грамматическим особенностям рассматриваемого вводного предложения. Слова «обязательных» компонентов в нём расположены в разном порядке. Последовательность «подлежащее $\boldsymbol{y}+$ сказуемое prey + дополнение ре» видим во вводной структуре предложения Dere leman, $\boldsymbol{y}$ prey $\boldsymbol{p} \boldsymbol{e}$ Pi wille Patow tel to me. (“The Romance of Guy Warwick", p. 64). Оборот, употреблённый в предложении And if ye wol nat so, my lady swete, Than preye I thee, to-morwe with a spere That Arcita me thurgh the herte bere. (Chaucer G., "The Knightes Tale", s. 2253), имеет другой порядок слов, глагол ргеуе выносится на первое место, за ним следует подлежащее и дополнение. Дополнение the размещается после подлежащего вводного оборота в предложении Speke no sich wordis, son, I the pray. ("Abraham. The Towneley Plays", p. 46).

Количество слов во вводном предложении не постоянно. “Обязательные” компоненты могут претерпевать редукцию, но также могут принимать и дополнительные элементы.

Если «обязательные» слова элиминируются, то это происходит только с местоимением-дополнением. Пример потери дополнения видим во вводном предложении структуры Honoureth hir, and loveth hir, I preye, Who-so me loveth; ther is na-more to seye (Chaucer G., "The Clerkes Tale", s. 370).

В ряде случаев состав маркера расширяется. В предложении And specially, here I the pray, To spede the as fast as thou may (“Caesar Augustus. The Towneley Plays", p. 82) наблюдаем оборот, в котором в качестве дополнительного элемента использовано наречие here, поставленное в препозицию к “обязательным” компонентам. В составе вводного оборота предложения And therfor, sir, I wold the pray, Thy best counsel thou wold me say ("Caesar Augustus. The Towneley Plays", р. 83) видим модальный глагол wold, размещённый между подлежащим и дополнением. Все приведённые выше предложения содержат маркеры, в которых после глагола pray следует беспредложное дополнение. Употребление предложного дополнения с образованием сочетания pray to thee является особенностью, которой характеризуется вводная клаузема в конструкции Telle me thyn hert I pray to the ("Abraham and Isaac", p. 54).

Далее рассмотрим расположение оборота в принимающем предложении. В том случае, если I pray yow / thee является единственным прагматическим маркером в предложении (исходим из 
пунктуационной разметки текстов в цитируемых изданиях и считаем знаком, маркирующим конец предложения, точку), то наиболее характерно его расположение в начале и финале, как в примерах: Tel on thy tale, maunciple, I thee preye (Chaucer G., "The Maunciples Tale", s. 103) и I pray Pe, dame, be stille ("The Fysshers and Marynars. York Plays", p. 49).

Расположение внутри принимающего предложения, как в примере But tak it not, I prey yow, in desdeyn (Chaucer G., “The Prologue”, s. 790), является гораздо менее частотным. Здесь прагматический маркер отделяет обстоятельство in desdeyn (“пренебрежительно, презрительно”) от главных членов предложения и выполняет в тексте, с одной стороны, функцию смягчения императива, и, с другой стороны, увеличивает объём строфы для рифмования между собой слов desdeyn и pleyn:

But tak it not, I prey yow, in desdeyn;

This is the poynt, to speken short and pleyn.

Если в предложении, помимо I pray yow / thee, употреблены и другие маркеры, предикативные или непредикативные, то их расположение в конструкции тяготеет к трём основным вариантам.

Первый вариант размещения маркеров назовём «рамочным». В этом случае маркеры располагаются в начале и финале предложения, образуя своеобразное фатическое обрамление для информативной части предложения. Так, структура Loo! here at hande Come hedir faste, dame, I Pe praye ("The Fysshers and Marynars. York Plays", p. 47) начинается междометием lоо и завершается комбинацией обращения dame и оборота I Ре praye. Если маркеров в начале предложения несколько, то первым обычно стоит непредикативный, как союз and в примере And therforee, if that I misspoke or seye, Wyte it the ale of Southwerk, I yow preye (Chaucer G., "The Knightes Tale", s. 3140), рядом находится ещё один непредикативный маркер, наречие therforee, завершает конструкцию оборот I yow preye. В отрывке "No fors," quod he, "now, sir, for goddes sake, What shal I paye? telleth me, I preye.” (Chaucer G., "The Chanouns Yemannes Tale”, s. 1357) наблюдаем комбинацию трёх маркеров - наречия now, обращения sir и оборота for goddes sake, - они образуют начало предложения, а две предикативные структуры, императив telleth mе и сочетание I preye размещены в финале. В следующем отрывке видим "рамочное» размещение трёх маркеров, наречия Nowe, оборота I Pe pray и существительного-обращения Isaak, которые образуют окружение императивного предложения kysse me hartely:

Nowe kysse me hartely, I Pe pray,

Isaak, I take my leue for ay (“The Parchemyners and Bokebynders. York Plays", p. 63).

Приведённые далее два варианта расположения прагматических маркеров в предложении назовем «точечными», оба они предполагают размещение маркеров в виде финальной или начальной группировки.

Концентрацию маркеров в финале предложения наблюдаем в примере What is Magnesia, good sir, I yow preye? (Chaucer G., "The Chanouns Yemannes Tale”, s. 1457), где автор использовал обращение good sir и I yow preye при оформлении вопросительной структуры.

Сосредоточение всех маркеров в начале предложения иллюстрируется несколькими следующими примерами. В конструкции I pray ре, dame, be stille ("The Fysshers and Marynars. York Plays", p. 49) императив предваряется оборотом I pray Бе и обращением dame. Четыре маркера, среди которых видим три непредикативных (союз And и наречия here и specially) и предикативный I the pray, размещаются в начале предложения And specially, here I the pray, To spede the as fast as thou may ("Caesar Augustus. The Towneley Plays", p. 82). Ещё более разнообразен состав фатической части в предложении And therfor, sir, I wold the pray, Thy best counsel thou wold me say ("Caesar Augustus. The Towneley Plays", p. 83), где используются союз And, наречие therfor, обращение sir и предикативный оборот I wold the pray. Междометие Ha, обращение gode suster, наречие yet и две предикативные структуры I prey и Tell те входят в состав начальной группировки вопросительного предложения $\mathrm{Ha}$, gode suster, yet I prey Tell me, why ye be so besey And with these halters thus begone? (Gower J., "Confessio Amantis”, p. 48).

Одним из авторских приёмов является такое оформление прямой речи, при котором комментарий вклинивается между двумя прагматическим маркерами. Так например, в отрывке Sir knizt, he seyd, y prey Pe, Tel me Pi name and whenne tow be ("The Romance of Guy Warwick", 
p. 54) наблюдаем сочетание he seyd, размещённое между обращением Sir knizt и оборотом y prey Pe.

В рифмованном тексте для I pray yow/thee весьма характерным является расположение в конце строфы. Порядок «обязательных» составляющих в обороте при этом будет зависеть от рифмуемого слова.

Например, в отрывке -

'Sadok' seyd Sedyn 'lete me be

WiP gode loue y pray Pe;' (“The Romance of Guy Warwick”, p. 104)

завершающий строфу глагол be диктует такой порядок слов во вводном предложении, при котором дополнение Ре требуется поставить после глагола pray.

Намерение автора рифмовать слово after обусловливает иной порядок слов в обороте, с размещением дополнения ре перед глаголом praye:

Lizt luflych adoun and lenge, I pe praye,

And quat-so by wylle is we schal wyt after ("Sir Gawain and the Green Knight", s. 8).

Если изучаемая структура размещается автором в рифмованном тексте таким образом, что она не завершает строфу, то порядок слов обусловлен ритмом. В отрывке -

I wol don sacrifice, and fyres bete.

And if ye wol nat so, my lady swete,

Than preye I thee, to-morwe with a spere

That Arcita me thurgh the herte bere (Chaucer G., “The Knightes Tale”, s. 2253) видим пример расположения маркера в начале строфы. На второе, пятое и восьмое слово в строчке Than preye I thee to-morwe with a spere, в соответствии с требованием ритма, должно падать ударение, поэтому в обороте preye I thee глагол как обязательное ударное слово, в отличие от двух местоимений, которые обычно не имеют ударения, стоит на первом месте.

Сочетание I pray yow / thee может быть представлено в тексте в форме не только вводного, но и главного предложения. В последнем случае за оборотом следует that-придаточное. Такой вариант встретился нам в «Кентерберийских рассказах». В функции главного предложения видим I pray yow в структуре I preye yow that ye wol reeve on me ("The Milleres Tale", s. 3362). Аналогично наблюдаемому для вводной структуры, состав оборота может быть расширен. Так, в предложении I pray yow alle that ye nat yow greve ("The Reves Tale", s. 3911) расширение происходит посредством добавления в состав дополнения ещё одного местоимения alle. Так же, как и вводная структура, образующий главное предложение оборот может комбинироваться с другими прагматическим маркерами. Совместное расположение preye I thee и наречия than в начале предложения иллюстрируется примером Than preye I thee, to-morwe with a spere That Arcita me thurgh the herte bere (“The Knightes Tale”, s. 2253).

\section{Заключение}

При изучении ряда структурных и стилистических характеристик прагматических маркеров, построенных глаголом pray и функционирующих в предложениях реплик персонажей в произведения XIV-XV вв., были отмечены следующие особенности.

Различия диалектов, на которых записывались произведения, отсутствие устоявшихся грамматических норм, а, возможно, и различное произношение составляющих оборот слов, обусловливает разницу в графическом отражении трёх основных компонентов оборота - подлежащего (I или Y), сказуемого (pray, praye, prey, preye), дополнения (yow, thee, the, pe).

Сочетание «подлежащее + сказуемое + дополнение» может быть принято за «обязательное», поскольку прослеживается тенденция к сохранению этих трёх слов в составе оборота.

На рассматриваемом историческом этапе оборот не является устоявшейся структурой. Состав оборота расширяется (за счёт наречия here I the pray, модального глагола I wold the pray, предлога I pray to the) или сужается (при редукции местоимения, служащего дополнением I preye). Порядок слов в обороте изменяется в зависимости от требований рифмы или предпочтения автора (I pe 
praye, y pray Pe, preye I thee). В предложении авторы оформляют I pray yow / thee не только как вводную клаузему, но и как главную, принимающую that-придаточную.

Расположение оборота относительно принимающего предложения зависит от того, является ли I pray yow / thee единственным прагматическим маркером или он скомбинирован с другими маркерами, предикативными, или непредикативными. Будучи единственным маркером в предложении, оборот преимущественно размещается в начале или финале конструкции, вклинивание в принимающее предложение не характерно и является индивидуальным выбором автора. При сочетании оборота с другими маркерами наблюдаются два варианта их размещения в предложении: «точечно», с концентрацией нескольких маркеров в начале или финале конструкции, и «рамочно», с размещением фатических составляющих в пре- и постпозиции от информативной части предложения. С конструкцией I pray yow / thee комбинируются обращения (dame, sir, good sir, gode suster, son), союзы (and), наречия (nowe, specially, than (вариант then), therfor), модальнопредикативные фатические структуры, представленные императивом (telleth me, tell me), устоявшиеся сочетания (for goddess sake).

(C) Меркурьева Н.Ю., 2021

\section{Источники иллюстративного материала}

1. Chaucer Geoffrey. The complete works of Geoffrey Chaucer. V. 4. Oxford: at the Clarendon Press, 1900. 667 p.

2. Gower John. Confessio Amantis. London: Bell and Daldy Fleet Street, 1857. 396 p.

3. Abraham and Isaac. The N-Town Play. Early English Text Society. S.S.11. Oxford: Oxford University Press, 1991. 413 p.

4. The Romance of Guy of Warwick. Early English Text Society. London: Trübner, 1883. 674 p.

5. Sir Gawain and the Green Knight. Oxford: Clarendon Press, 1967. 69 p.

6. Caesar Augustus. The Towneley Plays. London: Published for the Early English Text Society by K. Paul, Trench, Trübner, 1897. $460 \mathrm{c}$.

7. The Fysshers and Marynars. York Plays. Oxford: At the Clarendon Press, 1885. 269 p.

8. The Parchemyners and Bokebynders. York Plays. Oxford: At the Clarendon Press, 1885. 269 p.

\section{Список литературы}

1. Аракин В. Д. История английского языка / В. Д. Аракин. М.: Физматлит, 2009. 304 с.

2. Аракин В. Д. Очерки по истории английского языка / В. Д. Аракин. М.: Физматлит, 2007. 288 с.

3. Большой англо-русский словарь под общим руководством И. Р. Гальперина. Т. 2. Москва: Русский Язык, 1988.1072 с.

4. Власян Г.Р. Особенности функционирования маркеров англоязычного конверсационного дискурса / Г. Р. Власян. // Вестник Иркутского государственного лингвистического университета. 2011. № 4. С. 115-120.

5. Зайдес К.Д. Прагматические маркеры предикативного типа в устной спонтанной речи: подходы к описанию / К. Д. Зайдес. // Коммуникативные исследования. 2019. Т. 6. № 2. С. 375-396.

6. Иванова, И. П., Чахоян, Л.П., Беляева, Т.М. История английского языка / И. П. Иванова, Л. П. Чахоян, Т. М. Беляева. СПб.: Изд-во «Лань», 1999. 512 с.

7. Мюллер В. К. Англо-русский словарь / В. К. Мюллер. Москва: Русский Язык, 1982. 887 с.

8. Никола М. И. Английская литература XIV века: становление поэзии и прозы, истоки традиций. Автореф. дис. ...докт. филол. наук: 10.01.05 / Никола Мария Ивановна; Моск. пед. гос. ун-т. Москва, 1995. 23 с.

9. Online Etymology Dictionary. https://www.etymonline.com. (дата обращения 10.03.2021)

10. Смирницкий А. И. Лекции по истории английского языка / А. И. Смирницкий М.: Добросвет, 2006. 236 с.

11. Шустова С.В. Прагматический потенциал дискурсивных маркеров. / С.В.Шустова, Н.П. Царенко. // Теоретическая и прикладная лингвистика. 2019. Т. 5. № 4. С. 163-170.

12. Ярцева В.Н.Развитие национального литературного английского языка / В. Н. Ярцева. М.: Наука, 1969. 286 с.

13. Fraser B. What are discourse markers? // Journal of Pragmatics. 1999. № 31. P. 931-952.

14. Gonzales M. Pragmatic Markers and Discourse Coherence Relations in English and Catalan Oral Narrative // Discourse Studies. 2005. № 77(1). P. 53-87.

15. Mueller S. Discourse Markers in Native and Non-native English Discourse. Amsterdam: J. Benjamins, 2005. 290 p.

16. The Oxford History of English. Ed. by L. Mugglestone. Oxford: Oxford University Press, 2006. 485 p.

17. Shiffrin D. Discourse Markers / D. Schiffrin. Cambridge: Cambridge University Press, 1987. 364 p.

18. Verdonik D., Rojc M. et al. Annotating Discourse Markers in Spontaneous Speech Corpora on an Example for the Slovenian Language // Language Resources and Evaluation. 2007. V. 41. № 2. P. 147-180. 


\section{References}

1. Arakin, V.D. Istoriia angliiskogo iazyka [A history of the English language] / V.D. Arakin. Moscow: Fizmatlit, 2009. 304 p.

2. Arakin, V.D. Ocherki po istorii angliiskogo iazyka [Essays in the history of the English language] / V.D. Arakin. Moscow: Fizmatlit, 2007. 288 p.

3. Bolshoi anglo-russkii slovar pod obshchim rukovodstvom I.R. Galperina. V. 2. [English-Russian Dictionary under the guidance of I.R. Galperin]. Moscow: Russkii Iazyk, 1988. 1072 p.

4. Vlasian, G.R. Osobennosti funktsionirovaniia markerov angloiazychnogo konversatsionnogo diskursa [Functioning of markers of English conversational discourse] / Vlasian G.R. // Vestnik Irkutskogo gosudarstvennogo lingvisticheskogo universiteta [Irkutsk State Linguistic University Bulletin ]. 2011, 4, P. 115-120.

5. Zaides, K.D. Pragmaticheskie markery predikativnogo tipa v ustnoi spontannoi rechi: podkhody k opisaniiu. [Approaches to the description of predicative pragmatic markers in opal spontaneous speech] / K.D. Zaides // Communikativnye issledovaniia [Communication Studies]. 2019, 6 (2), P. 375-396.

6. Ivanova, I.P., Chakhoian L.P. et al. Istoriia Angliiskogo iazyka [The History of the English Language] / I.P. Ivanova, L.P. Chakhoian, T.M. Beliaeva. Saint-Petersburg: Lan, 1999. 512 p.

7. Miuller, V.K. Anglo-russkii slovar [Russian-English Dictionary] / V.K. Miuller. Moscow: Russkii Iazyk, 1982. P. 887 p.

8. Nickola, M.I. Angliiskaia literatura XIV veka: stanovlenie poezii I prozy, istoki traditsii [English literature of XIV century: formation of poetry and prose, origins of traditions]. PhD dissertation abstract / M.I. Nickola. Moscow Stage Pedagogical University: Moscow, 1995. 23 p.

9. Online Etymology Dictionary. Available at: https://www.etymonline.com. (Accessed 10 March 2021)

10. Smirnitskii, A.I. Lektsii po istorii angliiskogo iazyka. [Lectures on the English language history] / A.I. Smirnitskii. Moscow: Dobrosvet, 2006. 236 p.

11. Shustova, S.V., Tsarenko, N.M. Pragmaticheskii potentsial diskursivnykh markerov [Pragmatic potential of discourse markers] / S.V. Shustova, N.M. Tsarenko // Teoreticheskaia i prikladnaia lingvistika [Theoretical and Applied Linguistics]. 2019, 5 (4), P. 163-170.

12. Iartseva, V. N. Razvitie natsional'nogo literaturnogo angliiskogo iazyka [The development of national literary language]/ V.N. Iartseva. Moscow: Nauka, 1969. 286 p.

13. Fraser, B. What are discourse markers? // Journal of Pragmatics. 1999. № 31. P. 931-952.

14. Gonzales M. Pragmatic Markers and Discourse Coherence Relations in English and Catalan Oral Narrative // Discourse Studies. 2005. № 77(1). P. 53-87.

15. Mueller, S. Discourse Markers in Native and Non-native English Discourse. Amsterdam: J. Benjamins, 2005. 290 p.

16. The Oxford History of English. Ed. by L. Mugglestone. Oxford: Oxford University Press, 2006. 485 p.

17. Shiffrin, D. Discourse Markers. Cambridge: Cambridge University Press, 1987. 364 p.

18. Verdonik, D., Rojc, M., Stabej, M. Annotating Discourse Markers in Spontaneous Speech Corpora on an Example for the Slovenian Language // Language Resources and Evaluation. 2007. V. 41. № 2. P. 147-180.

\section{Сведения об авторе:}

Меркурьева Наталья Юрьевна - кандидат химических наук, доцент кафедры юриспруденции МГУУ ПМ им. Ю.М. Лужкова. Область научных и профессиональных интересов: теоретическая грамматика, грамматика разговорной диалогической речи, исторический синтаксис английского языка.

E-mail nata2lya1@mail.ru.

\section{About the author:}

Merkuryeva, Natalia - PhD, Associate Professor of Moscow Metropolitan Governance Yury Luzhkov University (Moscow, Russia). Spheres of research interest: theoretical grammar, spoken language grammar, historical syntax. E-mail: nata2lya1@mail.ru 